\title{
Research On Key Technology For The Development Of Smart Cities
}

\author{
Jia Baoxian ${ }^{1, a}$ \\ ${ }^{1}$ School of Computer Science of Liaocheng University \\ ajiabaoxian1221@163.com
}

Keywords: Smart City; Big Data; Data Ming; Semantic Search; Security protocol ;Harmony Search Algorithm;

Abstract: Currently, how to innovative use of big data becomes hot issues.Cloud computing, a new generation of information processing technology to examine the key issues in the development of modern cities, especially by means of data processing, data completely solve the problem that need to solve in the urban construction. Technology is the key technology to solve the problems of the city. We mainly introduced the key technology of space data, intelligent optimization algorithm, networking in the form of security protocol, and proposed effective solutions to these techniques, to provide technical support for the development of smart cities.

\section{Introduction}

Smart city is a new generation using information technology to perception, monitoring, analysis, integration of urban digital resources, to make a smart response to various needs, to create a green, harmonious environment for the public, to provide universal, convenient and efficient service of urban form. Smart city is the inevitable product of human society to the information society. It is a new concept and mode of the development of the world.

Smart city in China is a high level of information management and management of the city, the city's management to provide a large data for the city's running. While the city operation management big data is easy to become a significant target of network attacks, it lead to the city management information leak.

\section{Big space data research}

Concept.As the Internet, mobile Internet and cloud computing technology and application of booming development, human produce not only the amount of data with exponential growth, and the structure of the data become increasingly complex, beyond the traditional database management ability. Big Data Problem (Big Data Problem) become the focus of academia and industry information technology heat theory, data-centric paradigm - the era of "Big Data" has become the mainstream of information technology. Big data (big data) is massive (volume), a variety (variety), high-speed change (velocity), the true essence of complex data set (veracity) in these data, spatial data accounted for the vast majority, about $80 \%$ of the data related to the spatial location. Spatial data is the foundation of the people through information world understanding real world data source and wisdom.

Research Situation.In the industry, Microsoft (Microsoft) launched in 2011 with Windows compatible Big Data solutions based on Hadoop (Big Data Solution), as part of the SQL Server 2012 version. IBM provides InfoSphere BigInsights, not only the organic combination of DB2 and no database. Oracle database (Oracle) no (and Big Data Appliance combination, make customers have the ability to deal with unstructured Data directly. Google Bigtable mass structured data distributed storage, use BigQuery SQL query data. In addition, Apple's ripped, Facebook's The Open Compute Project, EMC Greenplum HD information such as The industry is also committed to provide large data solutions and applications. Baidu in China since 2007 using Hadoop do offline processing, 80\% Hadoop cluster are used for log processing, more than 10000 Hadoop server has gone beyond the Yahoo and Facebook, planned for 2013 to 20000, a day of data processing for 6 TB. In addition to baidu search log analysis, tencent and taobao and alipay, such as data warehouse also made the Hadoop processing mass data. 
Major technologies involved in large space data.Spatial data index technology. Because of the universality and importance of spatial data index, a large number of domestic and foreign scholars have carried out in-depth research. Many kinds of spatial data indexing techniques have been obtained. The spatial data index technology is composed of B tree index, two fork tree index, spatial object ranking method and Hashi index technology.

In the research of smart city, a large amount of spatial data will be generated, which will be an important application method. However, due to the large amount of data, the efficiency of query will be greatly reduced.

How to extract useful knowledge from the large data generated from the application of the smart city in China is the focus of the research of spatial data. The spatial data can be found by using the method of spatial analysis, statistical analysis, genetic algorithm, neural network, data visualization method. Can be used to study a set of space for the application and the wisdom of the city, efficient and high accuracy

Intelligent Optimization Scheduling Technology.Intelligent optimization scheduling is the main research content of intelligent city information processing and security. It can combine the optimization theory and the intelligent algorithm, which is applied to the practical problems such as task scheduling, intelligent transportation scheduling, intelligent logistics scheduling, and so on.

\section{Research Status.}

In recent years, intelligent algorithm is the main method to solve the scheduling problem [1-8], common intelligent algorithms including genetic algorithm, differential evolution, decentralized search, simulated annealing, variable neighborhood search, tabu search, ant colony algorithm, particle swarm optimization, iterative greedy algorithm, etc., which is the most widely used. However, the intelligent algorithm is based on the simple simulation of natural phenomena, or based on the improvement of local search method, which is lack of systematic and rigorous theoretical basis. Therefore, the optimization performance of a single intelligent algorithm is not high. The use of different optimization mechanism, optimization behavior and optimization of the structure of the complementary to improve the performance, the hybrid use of multiple algorithms to become the important means to solve the scheduling problem. In the scheduling algorithm, the algorithm improves the performance of the algorithm by combining the intelligent algorithm with the feature information and the heuristic rule. But from the research situation of intelligent optimization scheduling problem, the research on the problem of feature information and its combination with the intelligent algorithm needs to be further deepened.

\section{Harmony Optimization Algorithm.}

With the development of Intelligent City, the optimization technology has gradually become an important research topic, which can be used to solve many practical engineering problems, the purpose is to find the best solution from many engineering problems. The process of harmony algorithm can be summarized as following five steps:

1: Step initialization optimization problem and algorithm parameters. We use the minimization problem to explain the optimization problem:

The solution vector is the decision variable, which is the lower and upper limit of the decision variables, and the number of decision variables is the number of decision variables. In addition, the initialization of the parameters of the harmony search algorithm, including, as well as the algorithm termination conditions.

Initialization of

2: HM Step. Randomly generate a harmony into the harmony, and the form of the harmony library is as follows:

The decision variable in HM, which is generated by the random number assignment within the inner. Can be obtained by the next type of the first solution vector variables: 
3: Step to produce a new harmony. In the process of improvisation, a new harmonic vector is generated by three rules: 1) memory library selection; 2). In the process of selecting the memory database, the decision variable of the new vector is obtained from the decision variable in the memory bank, and the probability of selecting a new vector is randomly selected from the feasible region.

The variable values obtained from each passing probability will further determine if the tone is required to be fine tuned. Which represents the execution probability of the process, indicating that the bandwidth is adjusted to be a preset value, is a random number between 0 to 1 .

4: Step update. According to the objective function value, if the new harmony vector is better than the worst vector, then the new vector is replaced by the most poor harmony vector, otherwise it will not operate. This is actually the choice of algorithm, and the objective function is to measure whether the new vector is stored in the memory database.

5: Step check if the stop condition is reached. If the stop condition is reached, the algorithm terminates, otherwise repeat step 3-4.

Through the research, the hybrid intelligent scheduling theory and method based on the new group optimization algorithm is established, and it is applied to the problem of intelligent city information processing. The research results will enrich and deepen the existing optimization scheduling theory, solve the problem of urban information processing, has a broad application prospects, significantly improve the efficiency of urban operation and management.

\section{Concluding Remarks}

The research of the above problems is the main work of the research on the information processing and security of China's smart city. Technology is the key technology to effectively solve the above problems. It is based on information, knowledge and intellectual resources. Through a transparent, full of information access, wide and safe information transmission, effective and scientific information processing, balance and effectively improve the urban transportation and management efficiency, improve the urban public service level, so as to improve the urban development of innovative, orderly and sustainable, forming a low carbon city ecological circle, the new form of urban development.

\section{References}

[1] Biskup D. A state-of-the-art review on scheduling with learning effects [J]. European Journal of Operational Research, 2008, 188(2):315-329.

[2] Qian B, Wang L, Huang DX, Wang X. An effective hybrid DE-based algorithm for flow shop scheduling with limited buffers [J]. International Journal of Production Research 2007, doi: 10.1080/00207540701528750.

[3] Li BB, Wang L. A hybrid quantum-inspired genetic algorithm for multiobjective flow shop scheduling [J]. IEEE Transactions on Systems Man and Cybernetics Part B, 2007, 37:576-591.

[4] Ruiz R, Stutzle T. A simple and effective iterated greedy algorithm for the permutation flowshop scheduling problem [J]. European Journal of Operation Research, 2007, 177(3): 2033-2049.

[5] Pan Q K, Tasgetiren M F, Liang Y C. Minimizing Total Earliness and Tardiness Penalties with a Common Due Date on a Single-Machine Using a Discrete Particle Swarm Optimization Algorithm [J]. Lecture notes in computer science, 2006, 4150:460-467.

[6] Pan QK, Tasgetiren MF, Liang YC. A Discrete Particle Swarm Optimization Algorithm for the No-Wait Flowshop Scheduling Problem [J]. Computers and Operations Research, 2007, doi: 10.1016/j.cor.2006.12030.

[7] Geiger M J. On operators and search space topology in multi-objective flow shop scheduling [J]. European Journal of Operational Research, 2007, 181(1):195-206.

[8] Lowe G.Breaking and fixing the Needham-Schroeder publickey protocol using FDR[J].Software-Concepts and Tools,1996(17):93-102 